\title{
The effects of xanthine oxidase inhibitor in patients with chronic heart failure complicated with hyperuricemia: a prospective randomized controlled clinical trial of topiroxostat vs allopurinol-study protocol
}

\author{
Masashi Sakuma ${ }^{1} \cdot$ Shigeru Toyoda $^{1} \cdot$ Takuo Arikawa $^{1} \cdot$ Yota Koyabu $^{1} \cdot$ Toru Kato $^{2} \cdot$ Taichi Adachi $^{3} \cdot$ Hideaki Suwa $^{3}$. \\ Jun-ichi Narita ${ }^{4} \cdot$ Koetsu Anraku $^{5} \cdot$ Kimihiko Ishimura $^{5} \cdot$ Fumitake Yamauchi $^{6} \cdot$ Yasunori Sato $^{7} \cdot$ Teruo Inoue $^{1} \cdot$ For \\ Excited UA study Investigators
}

Received: 2 March 2018 / Accepted: 1 June 2018 / Published online: 18 June 2018

(c) The Author(s) 2018

\begin{abstract}
Background Hyperuricemia has a close relationship with cardiovascular diseases including heart failure. However, it is controversial whether xanthine oxidase inhibition has benefits for patients with chronic heart failure. We designed the Effect of Xanthine Oxidase Inhibitor in Chronic Heart Failure Patients Complicated with Hyperuricemia study (Excited-UA study) to compare the beneficial effects between a novel xanthine oxidoreductase inhibitor, topiroxostat, and a conventional agent, allopurinol, in patients with chronic heart failure and hyperuricemia. We focus on serum N-terminal pro-brain natriuretic peptide (NT-proBNP) level, echocardiography-based cardiac function, vascular endothelial function, renal function, inflammation, and oxidative stress.

Methods The excited-UA is a prospective, randomized, open-label, blinded-endpoint clinical trial designed to prove our hypothesis that topiroxostat is more effective than allopurinol in patients with chronic heart failure and hyperuricemia. A total of 140 patients with chronic heart failure and hyperuricemia (plasma brain natriuretic peptide level $\geq 40 \mathrm{pg} / \mathrm{mL}$ and serum uric acid level $\geq 7.0 \mathrm{mg} / \mathrm{dL}$ ) are randomly assigned (ratio 1:1) into either the topiroxostat group (40-160 mg/day) or allopurinol group (100-300 mg/day), to achieve the target uric acid level of $6.0 \mathrm{mg} / \mathrm{dL}$. According to the protocol, all patients are followed up annually for 24 weeks. The primary endpoint is percent change in serum NT-proBNP level at 24 weeks from baseline.

Conclusions The Excited-UA study would provide novel evidence for the clinical relevancy of xanthine oxidoreductase inhibitor treatment in patients with chronic heart failure and hyperuricemia.
\end{abstract}

Keywords Allopurinol $\cdot$ Chronic heart failure $\cdot$ Hyperuricemia $\cdot$ N-terminal pro-brain natriuretic peptide (NT-proBNP) . Topiroxostat $\cdot$ Xanthine oxidase inhibitor

\section{Introduction}

Hyperuricemia has a close relationship with cardiovascular diseases and is an independent risk factor for these diseases $[1,2]$. There is considerable evidence showing that an increased serum level of uric acid is an independent

Electronic supplementary material The online version of this article (https://doi.org/10.1007/s10157-018-1599-6) contains supplementary material, which is available to authorized users.

Teruo Inoue

inouet@dokkyomed.ac.jp

Extended author information available on the last page of the article atherogenic risk factor [3]. In addition to atherosclerotic risk, hyperuricemia has been a focus regarding its relationship with heart failure. The incidence of hyperuricemia is high in patients with chronic heart failure and is a predictive factor for prognosis in these patients $[4,5]$. Thus, uricemic control would be an important target to treat in patients with chronic heart failure and hyperuricemia. Nevertheless, it is still controversial whether xanthine oxidase inhibition has benefits in these patients.

A novel nonpurine selective inhibitor of xanthine oxidoreductase, topiroxostat, which has been developed as a potential alternative to a conventional xanthine oxidoreductase inhibitor, allopurinol, effectively reduces serum uric acid level in patients with hyperuricemia with or without 
gout. Unlike allopurinol, topiroxostat has shown renoprotective effects as demonstrated by the reduction of urinary albumin excretion, and thus can also be used without dose reduction in patients with mild to moderate renal dysfunction [6]. In addition, it is also suggested that topiroxostat ameliorates impaired vascular endothelial function beyond the uric acid lowering action [7]. These pharmacological actions of topiroxostat lead us to the hypothesis that this drug could have superior effects to allopurinol for patients with chronic heart failure and hyperuricemia.

We designed the Effect of Xanthine Oxidase Inhibitor in Chronic Heart Failure Patients Complicated with Hyperuricemia study (Excited-UA study) to investigate the beneficial effects of topiroxostat for patients with chronic heart failure and hyperuricemia, and compare them with those of allopurinol. We focused on serum $\mathrm{N}$-terminal pro-brain natriuretic peptide (NT-proBNP) level, echocardiographybased cardiac function, vascular endothelial function, renal function, inflammation, and oxidative stress.

\section{Methods}

\section{Study overview and design}

The Excited-UA is a prospective, randomized, open-label, blinded-end-point clinical trial designed to prove our hypothesis that topiroxostat is more effective in patients with chronic heart failure and hyperuricemia than allopurinol, using serum NT-proBNP level as a landmark. Eligible patients, recruited from 4 centers (Dokkyo Medical
University Hospital, National Hospital Organization Tochigi Medical Center, Tochigi Medical Center Tochinoki, and Yuai Memorial Hospital) are randomly assigned (ratio 1:1) into either the topiroxostat group (40-160 mg/day) or allopurinol group (100-300 mg/day) to achieve the target uric acid level of $6.0 \mathrm{mg} / \mathrm{dL}$. According to the protocol, all patients are followed up annually for 24 weeks. Trial design is shown in Fig. 1.

\section{Study population}

A total of 140 patients with chronic heart failure and hyperuricemia are scheduled to be enrolled from December 2015 to April 2018 and will be followed-up for 24 weeks. Prior to the assessment of eligibility, every participant is required to receive an adequate explanation based on the study plan and written informed consent is obtained from each patient. Detailed inclusion and exclusion criteria are listed in Table 1. In brief, eligible patients are aged $\geq 20$ and $<85$ years and have chronic heart failure, defined as plasma brain natriuretic peptide (BNP) level $\geq 40 \mathrm{pg} / \mathrm{mL}$ [8], and hyperuricemia, defined as serum uric acid level $\geq 7.0 \mathrm{mg} /$ $\mathrm{dL}$ [9] and receiving no antihyperuricemic agents within 4 weeks of participation.

\section{Registration and randomization}

In this study, registration and randomization of the patients are performed using the electric data capturing (EDC) system. The investigators input the patient information necessary for randomization into the EDC system, and then

Table 1 Inclusion and exclusion criteria

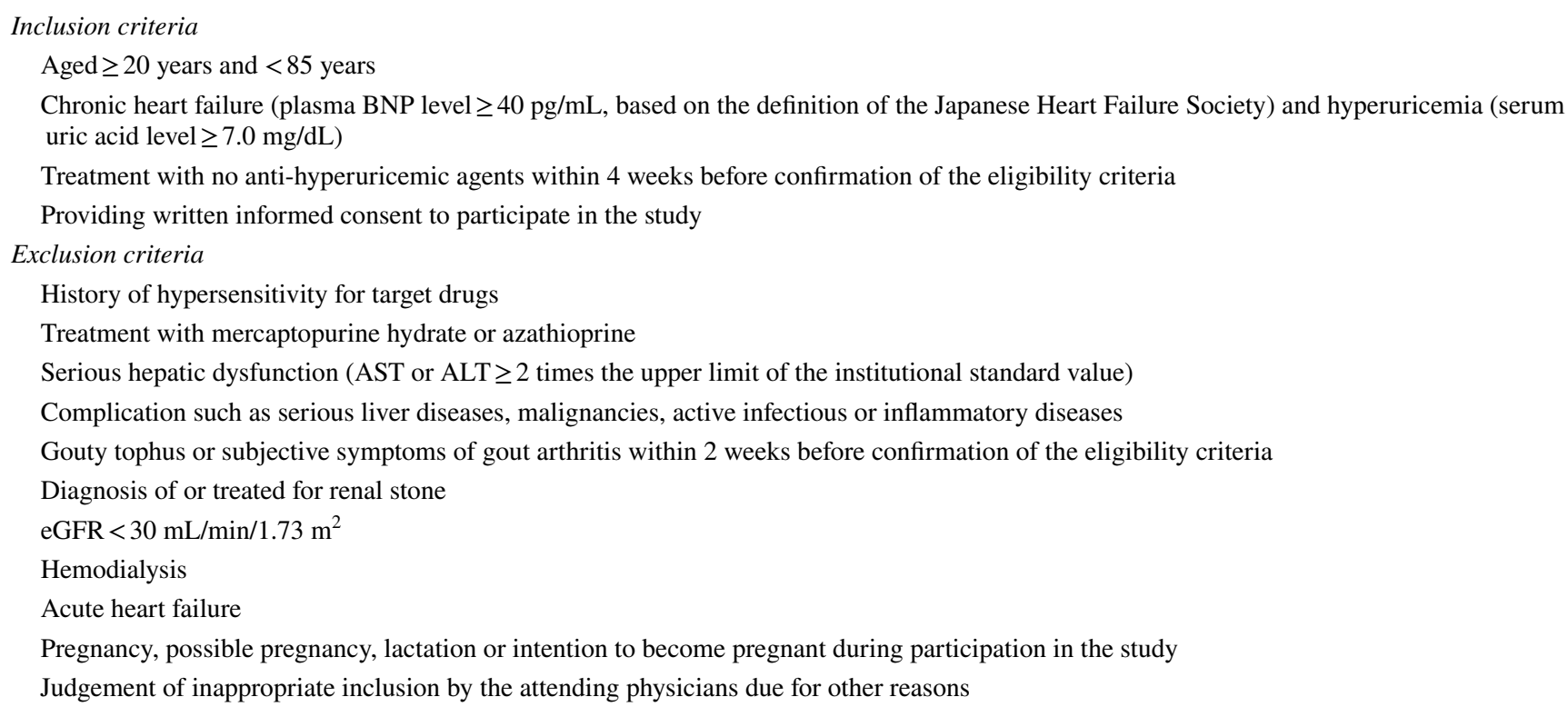

$B N P$ brain natriuretic peptide, $A S T$ aspartate transaminase, $A L T$ alanine aminotransferase, $e G F R$ estimated glomerular filtration rate 
confirmed randomization results and central registration numbers to discriminate the study participants. Randomization is performed using a minimization method with a biased-coin assignment balancing on plasma BNP level at registration $(<200, \geq 200 \mathrm{pg} / \mathrm{mL})$ and echocardiographybased left ventricular ejection fraction $(<45, \geq 45 \%)$.

\section{Dose setting/prohibited drugs for combined use}

The usual daily dosage of topiroxostat and allopurinol are 40-160 and 100-300 mg. respectively, in Japan. Thus, participants assigned to the topiroxostat group receive topiroxostat at an initial dose of $40 \mathrm{mg} /$ day; the dose was increased to $160 \mathrm{mg}$ /day every 4 weeks to achieve the appropriate uric acid level of $6.0 \mathrm{mg} / \mathrm{dL}$. Patients assigned to the allopurinol group receive allopurinol at an initial dose of $100 \mathrm{mg} /$ day. Of these patients; patients with renal dysfunction (estimated glomerular filtration rate: eGFR $\leq 50 \mathrm{~mL} / \mathrm{min} / 1.73 \mathrm{~m}^{2}$ [10] or creatinine clearance: $\mathrm{CCr} \leq 50 \mathrm{~mL} / \mathrm{min}$ ) receive a fixed dose of $100 \mathrm{mg} / \mathrm{day}$ during the observation period. Conversely, in patients without renal dysfunction, the dose of allopurinol was increased to $300 \mathrm{mg} /$ day every 4 weeks to achieve the target uric acid level of $6.0 \mathrm{mg} / \mathrm{dL}$. If the uric acid level decreased to $\leq 2.0 \mathrm{mg} / \mathrm{dL}$ during the study interval, the dose of each agent is reduced to the previous dose $(20 \mathrm{mg} /$ day reduction in topiroxostat and $50 \mathrm{mg} /$ day reduction in allopurinol).

Prohibited concomitant drugs include allopurinol in the topiroxostat group and topiroxostat in the allopurinol group. In both groups, uricosuric drugs such as benzbromarone, probenecid, and bucolome, drugs for improving acidemia such as potassium citrates-sodium citrate hydrate, antituberculosis agents/ immunosuppressive agents potentially affecting uric acid level such as pyradinamide, ethambutol, mizoribine, and ciclosporin, and drugs potentially interacting with topiroxostat or allopurinol such as mercaptopurine azathioprine, xanthine agents, eplerenone. or sodium glucose co-transporter 2 inhibitors are also prohibited (Table 2).

\section{Observations/measurements}

Detailed items for observations or measurements are shown in additional file 1. Baseline characteristics are screened prior to randomization. Specific biomarkers as below were collectively measured in core laboratories. NT-proBNP determinations are performed within one run, using a Roche Diagnostic NT-proBNP electrochemiluminescent immunoassay kit on a Elecsys 2010 analyser (Roche Diagnostics, Mannheim, Germany) according to the manufacturer's recommendations. The intra-assay variability of the NT-proBNP test was 3.9\% [11]. Urinary
Table 2 Prohibited concomitant agents

\begin{tabular}{l}
\hline Anti-hyperuricemic agents \\
\hline Uricosuric agents (benzbromarone, probenecid, bucolome) \\
Xanthine oxidoreductase inhibitors \\
Topiroxostat group: allopurinol, febuxostat \\
Allopurinol group: topiroxostat, febuxostat \\
Antiaciduric agents (potassium citrate-sodium citrate hydrate) \\
Anti-tuberculosis agents potentially affecting serum uric acid level \\
Pyradinamide \\
Ethambutol \\
Immunosuppressive agents potentially affecting serum uric acid level \\
Mizoribine \\
Ciclosporin \\
Drugs possibly interacting with the target agents \\
Mercaptopurine, azathioprine \\
Xanthine agents (theophylline, etc) \\
Eplerenone \\
SGLT2 inhibitors
\end{tabular}

SGLT2 sodium glucose co-transporter 2

liver-type fatty acid binding protein (L-FABP) level and urinary albumin excretion are measured for the assessment of renal function. Oxidative stress is assessed by reactive oxygen metabolites (d-ROM) and biological anti-oxidant potential (BAP) tests as well as urinary 8-hydroxy-2'deoxyguanosine (8-OHdG) level. Multiple cytokine assay is conducted as per the manufacturer's instructions (Luminex Corp., Austin, TX), using a commercially available kit (BioSource International, Inc., Camarillo, CA). The results of these biomarkers are not disclosed until the end of follow-up period. Using echocardiography, we measure the following parameters: left ventricular ejection fraction (LVEF: modified Simpson method), left ventricular end-diastolic dimension (LVDd), left ventricular endsystolic dimension (LVDs), left atrial dimension (LAD), tricuspid pressure gradient (TRPG) inferior vena cava dimension (IVCD), peak early diastolic flow velocity $(E)$, E-wave deceleration time (DT), peak atrial systolic flow velocity $(A)$, early diastolic mitral annular velocity $\left(e^{\prime}\right)$, and the $E$ to $e^{\prime}$ ratio $\left(E / e^{\prime}\right)$. These parameters were evaluated by recording 3 cardiac cycles under stable conditions and the mean of the measurements was used for analysis. Vascular endothelial function is assessed by measurement of brachial artery flow-mediated dilation (FMD) ultrasonically using an automatic measurement system (UNEXEF 38G; Unex Co. Ltd., Nagoya, Japan) according to the standard protocol described in the guidelines [12] and Japanese guidelines of the Vascular Failure Working Group [13], and/or reactive hyperemia peripheral arterial tonometry (RH-PAT) using an EndoPAT 2000 device (Itamar Medical, Caesarea, Israel), as described previously [14]. 
Fig. 1 Trial design

Topiroxostat group

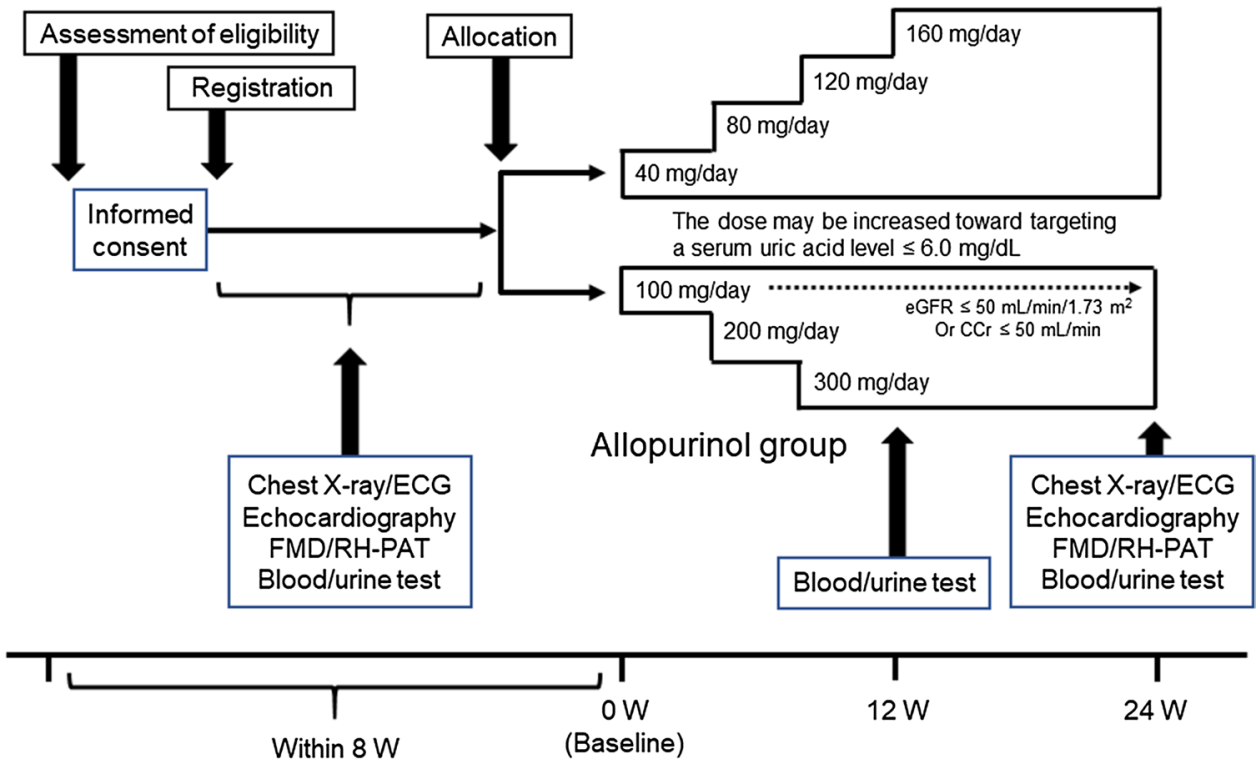

\section{Study endpoint}

The primary endpoint is percent change in serum NTproBNP level at 24 weeks from baseline. The secondary end points are as follows: (1) percent change in serum NTproBNP level at 12 weeks from baseline; (2) percent change in plasma BNP level at 12 and 24 weeks from baseline; (3) correlation between the percent change at 24 weeks in the NT-proBNP level and that in the BNP level; (4) change in FMD and RH-PAT values at 24 weeks from baseline; (5) correlation between the change amount at 24 weeks in the FMD value and that in the RH-PAT value; (6) change in serum uric acid level at 24 weeks from baseline; (7) change amounts and percent changes of specific biomarker values at 24 weeks from baseline; (8) change in echocardiographic parameter values at 24 weeks from baseline. In addition, as safety endpoints, we assess the frequency and proportion of adverse events such as gout arthritis and hepatic dysfunction.

\section{Data and safety monitoring}

The data and safety monitoring board blinded to study allocation also independently evaluate the safety of the whole trial. They assess the necessity for revision of trial design and the validity for continuance of trial entry, and recommend such issues to the chief investigator, if needed. The withdrawal or dropout criteria are listed in Table 3.

\section{Sample size determination}

A prior study demonstrated that topiroxostat treatment reduced plasma BNP level from $369.8 \pm 289.1$ to $224.4 \pm 141.9 \mathrm{pg} / \mathrm{mL}$ in patients with chronic heart failure and hyperuricemia [15], while several studies indicated that BNP or NT-proBNP did not change after allopurinol treatment [16-18]. In the present study, we converted the data of BNP values in these reports into the NT-proBNP values as reference values. Consequently, we assumed for percent change of NT-proBNP level that the average is $-39.0 \pm 75.0 \%$ in the topiroxostat group and assumed no change in the allopurinol group each in both groups. Based on these assumptions, the number of participants needed to detect statistical significance of topiroxostat is 60 patients per group to yield $80 \%$ power with a two-sided significance level of $5 \%$. In consideration of dropout, the target sample
Table 3 Withdrawal/dropout criteria

\footnotetext{
Hospitalization

Offer to discontinue or to retract agreement for participation by participants or their families

Considered inappropriate to continue the study by investigators due to aggravation of primary disease or complications

Considered inappropriate to continue the study by investigators due to adverse side effects of the target agents

Considered inappropriate to continue the study by investigators due to another reason

Hospital visits impossible due to moving or other reasons
} 
size was finally estimated to be a total of 140 patients, with 70 patients per group.

\section{Statistical analyses}

Statistical analyses and reporting of this trial will be conducted in accordance with the Consolidated Standards of Reporting Trials statement guidelines. The primary analyses performed in the full analysis set (FAS) include all participants except for the patients who rescind consent to the trial or are registered outside the contract period. For the baseline variables, summary statistics will be constructed using frequencies and proportions for categorical data, and means and standard deviations for continuous variables. Patient characteristics will be compared using Chi-square test or Fisher's exact test for categorical outcomes, and unpaired Student's $t$ test or Wilcoxon rank-sum test for continuous variables, as appropriate, between the two study groups. For the primary analysis comparing treatment effects between the treatment groups, the baseline-adjusted means and their 95\% confidence intervals will be estimated by analysis of covariance adjusted for the allocation factors, such as plasma BNP level $(<200, \geq 200 \mathrm{pg} / \mathrm{mL})$ and echocardiographybased left ventricular ejection fraction $(<45, \geq 45 \%)$ at the time of screening.

Regarding the analysis of primary endpoints, percent change in serum NT-proBNP level at 24 weeks from baseline is calculated for each group, and the difference between the two study groups is analyzed, using the unpaired $t$ test or Wilcoxon rank-sum test. The proportion of patients achieving at least 30\% reduction of NT-proBNP level is compared between the two study groups, based on a previous literature [19], using the Chi-square test or Fisher's exact test. Regarding the secondary endpoints, continuous variables are analyzed using paired $t$ test or Wilcoxon signed-rank test for intra-group comparison and inter-group comparisons and unpaired $t$ test or Wilcoxon rank-sum test for inter-group comparison. The Chi-square test (if necessary, Fisher's exact test) is performed to compare the categorical variables including frequency of adverse effects between groups. For correlation analyses, Pearson's product-moment correlation coefficient or Spearman's rank-correlation coefficient is calculated to assess its significance. All comparisons have been planned, and all $P$ values will be two-sided. $P$ values $<0.05$ will be considered statistically significant. All statistical analyses will be performed using SAS V.9.4, and are described in the statistical analysis plan, which will be fixed prior to database lock.

\section{Trial organization and oversight}

Details of trial organization are shown in Additional file 2. The principal investigators of the Excited-UA trial are Teruo
Inoue (chief investigator) and Masashi Sakuma (central committee member) of the Department of Cardiovascular Medicine, Dokkyo Medical University. The Steering Committee will carry out planning, operating, analyzing, and presentation of the trial. An independent data and safety monitoring board will evaluate safety during the study period. The trial secretariat is in a contact research organization (Soiken, Inc., Osaka, Japan). Data management, monitoring, statistical analyses, and audit will be independently implemented on the basis of outsourcing agreement.

\section{Study progress and current status}

The end of the recruitment period was initially set at October 2017; however, this has been extended to April 2018 due to a shortage of study participants. At present (3 November 2017), a total of 118 patients have been recruited into the study.

\section{Discussion}

The Excited-UA study is an ongoing, multicenter, prospective, randomized, investigator-initiated clinical trial aimed at testing the effects of treatment with topiroxostat for 24 weeks in patients with chronic heart failure and hyperuricemia, and compares them with those of allopurinol treatment for 24 weeks. The primary endpoint is percent change in serum NT-proBNP level at 24 weeks from baseline. The NT-proBNP level is collectively measured in a core laboratory and its values are not disclosed until the end of follow-up period, so the blinded-end-point fashion would be guaranteed.

It has been demonstrated that hyperuricemia has a close relationship with cardiovascular diseases, including chronic heart failure [1, 2]. Sakai et al. [20] demonstrated that hyperuricemia is a prognostic predictor independent of plasma BNP level in patients with chronic heart failure and that high level of the plasma uric acid was in part derived from secretion from the failing heart. Krishnan et al. [21] observed in the Framingham Offspring Cohort that serum uric acid level was associated with echocardiographic abnormalities representing left ventricular hypertrophy as well as left ventricular dysfunction. In addition, Fujimura et al. [22] observed that left ventricular hypertrophy, low left ventricular ejection fraction, and increased plasma BNP level were associated with plasma xanthine oxidoreductase level among various patients with heart disease. As mentioned above, growing evidence indicates that hyperuricemia and xanthine oxidoreductase is important in the risk of heart failure. Nevertheless, the effects of uric acid lowering therapy with xanthine oxidase inhibition for patients with heart failure are controversial. Erdogan et al. [23] observed that 3-month treatment 
with allopurinol was associated with improvement of coronary flow reserve and left ventricular function in patients with dilated cardiomyopathy and hyperuricemia. Xiao J et al. [24] demonstrated that 3-month allopurinol treatment resulted in an increase in FMD of the brachial artery and that echocardiographic cardiac function parameters improved after 6-month treatment in non-hyperuricemic patients with chronic heart failure. In contrast, the xanthine oxidase inhibition for patients with hyperuricemia and heart failure (EXACT-HF) study results indicated that allopurinol failed to improve clinical status, exercise capacity, quality of life, or left ventricular ejection fraction in high-risk patients with heart failure with reduced ejection fraction and elevated uric acid levels [20].

Recently, novel inhibitors of xanthine oxidoreductase such as febuxostat or topiroxostat have been developed. Compared to allopurinol, febuxostat can be administered to patients with mild to moderately impaired renal function because of its dual excretion pathway [25]. Febuxostat is widely used in patients with hyperuricemia and has superior anti-atherosclerotic effects, compared to allopurinol [26]. From such backgrounds, a study to evaluate the effects of febuxostat on ultrasonography-based intima-media thickness of the carotid artery, as a surrogate marker of cardiovascular disease risk, in patients with hyperuricemia is now ongoing [27]. Conversely, topiroxostat is characterized by its renoprotective effects as demonstrated by the reduction of urinary albumin excretion [6]. Kawamorita et al. [28] demonstrated that the renoprotective effects of topiroxostat could be attributed to its potential anti-oxidative action as demonstrated by inhibiting nicotinamide adenine dinucleotide phosphate (NADPH) oxidase in concert with suppression of intracellular uric acid production. In addition, it is also suggested that topiroxostat improves vascular endothelial function [7]. Recently, Kuki et al. [15] demonstrated that topiroxostat reduced not only serum uric acid level, but also urinary albumin excretion, serum hsCRP level, and plasma BNP level in patients with hyperuricemia at high risk for cardiovascular diseases. Our hypothesis that topiroxostat would be effective in chronic heart failure is based on these pleiotropic pharmacological actions, especially BNP reduction, of topiroxostat.

In the Excited-UA study, chronic heart failure and hyperuricemia were defined as a plasma BNP level $\geq 40 \mathrm{pg} / \mathrm{mL}$ and serum uric acid level $\geq 7.0 \mathrm{mg} / \mathrm{dL}$, respectively. Thus, this trial includes patients with very mild heart failure and those with very mild hyperuricemia, based on our hypothesis that early and aggressive intervention to hyperuricemia would prevent a progression of heart failure. In this trial, we measure various echocardiographic parameters including left ventricular diastolic as well as systolic functions to assess the effects of xanthine oxidoreductase inhibitors on cardiac function. It has been suggested that systemic inflammation, oxidative stress, and vascular endothelial dysfunction play a pivotal role in pathophysiology of heart failure [29-31]. If topiroxostat has anti-inflammatory, antioxidative, and vascular protective actions, it would provide beneficial effects in the pathophysiology of heart failure. Thus, in the Excited-UA trial, we measure serum levels of inflammatory biomarkers such as hsCRP and inflammatory cytokines. For assessment of oxidative stress state, the urine level of $8-\mathrm{OHdG}$ is measured, and d-ROM and BAP tests are performed. These biomarkers are assessed as the secondary endpoints. Chronic heart failure is often complicated with renal dysfunction. Existence of chronic kidney disease independently predict prognosis of heart failure. Cardiac and renal diseases share several common pathways, including inflammation, neuro-hormonal responses, metabolic and nutritional changes, and hemodynamic, acid-base or fluid statuses. Therefore, a concept as the cardio-renal syndrome has been proposed [32]. BNP and NT-proBNP are both established markers of prognosis and diagnosis in chronic heart failure. NT-proBNP is considered to be affected more by renal function than BNP [33], and, thus, may comprehensively reflect cardiac and renal functions. In the Excited-UA trial, we selected serum NT-proBNP level as a marker for the primary endpoint decision, although the definition of chronic heart failure in the patient inclusion criteria is based on plasma BNP level. Since topiroxostat has renoprotective effects, it would be rational that we assess both cardiac and renal effects in the pathophysiology of heart failure using NT-proBNP level, but not BNP level. In addition, BNP is affected in-vivo by degradation via neutral endopeptidase and endocytosis via clearance receptor, but NT-proBNP is not. Therefore, the NT-proBNP level shows longer half-life, and thus, may be more accurate biomarker for heart failure, compared with the BNP [34]. Actually, the NT-proBNP has been adopted as a study endpoint biomarker in a number of clinical trials for heart failure [19, 35]. In this trial, urine L-FABP level and urinary albumin excretion are simultaneously measured for the assessment of renal function. Finally, in the Excited-UA trial, we also assess xanthine oxidoreductase activity. It would also be promising to determine if the change in various echocardiographic parameters and biomarkers in the pathophysiology of heart failure after treatment with xanthine oxidoreductase inhibitors depends on xanthine oxidoreductase activity.

\section{Conclusions}

We designed the Excited-UA study to investigate the effects of topiroxostat for patients with chronic heart failure and hyperuricemia, and compare them with those of allopurinol. We focused on serum NT-proBNP level, echocardiographybased cardiac function, vascular endothelial function, renal 
function, inflammation, and oxidative stress. This trial would provide novel evidence for the clinical relevancy of xanthine oxidoreductase inhibitor treatment in patients with chronic heart failure and hyperuricemia.

Acknowledgements To conduct this trial, outsourcing agreement was signed between Dokkyo Medical University and Sanwa Kagaku Kenkyusho, Co., Ltd., Tokyo, Japan. The authors would like to thank all staff and patients who are participating in this trial, and also to thank Editage (http://www.editage.jp) for English language editing.

Author contributions All authors were involved in trial planning and operation. TI was responsible for drafting the article and preparing the figure and tables. The other authors critically supervised the whole article. YS carefully supervised the section on statistical analysis. All authors read and approved the final manuscript.

Funding This trial was financially sponsored by Sanwa Kagaku Kenkyusho, Co., Ltd.

\section{Compliance with ethical standards}

Conflict of interest The authors declare that they have no competing interests.

Ethics standards Prior to initiation, the study protocol needed to be approved by the local institutional review boards and independent ethics committees at every site. The trial is conducted in full compliance with the Declaration of Helsinki and according to the Ethical Guidelines for Medical and Health Research Involving Human Subjects established by the Ministry of Health, Labour, and Welfare. The information of this study was registered with University Hospital Medical Information Network clinical trial registry (No. UMIN000020939).

Open Access This article is distributed under the terms of the Creative Commons Attribution 4.0 International License (http://creativeco mmons.org/licenses/by/4.0/), which permits unrestricted use, distribution, and reproduction in any medium, provided you give appropriate credit to the original author(s) and the source, provide a link to the Creative Commons license, and indicate if changes were made.

\section{References}

1. Feig DI, Kang DH, Johnson RJ. Uric acid and cardiovascular risk. N Engl J Med. 2008;359:1811-21.

2. Li M, Hou W, Zhang X, Hu L, Tang Z. Hyperuricemia and risk of stroke: a systematic review and meta-analysis of prospective studies. Atherosclerosis. 2014;232:265 - 70.

3. Feig DI, Mazzali M, Kang DH, Nakagawa T, Price K, Kannelis J, Johnson RJ. Serum uric acid: a risk factor and a target for treatment? J Am Soc Nephrol. 2006;17:S69-73.

4. Anker SD, Doehner W, Rauchhaus M, et al. Uric acid and survival in chronic heart failure: validation and application in metabolic, functional, and hemodynamic staging. Circulation. 2003;107:1991-7.

5. Hamaguchi S, Furumoto T, Tsuchihashi-Makaya M, et al. Hyperuricemia predicts adverse outcomes in patients with heart failure. Int J Cardiol. 2011;151:143-7.

6. Hosoya T, Ohno I, Nomura S, et al. Effects of topiroxostat on the serum urate levels and urinary albumin excretion in hyperuricemic stage 3 chronic kidney disease patients with or without gout. Clin Exp Nephrol. 2014;18:876 - 84.

7. Tanaka A, Nakamura T, Sato E, Node K. Clinical effects of topiroxostat on renal and endothelial function in a patient with chronic kidney disease and hyperuricemic arteriopathy: a case report. Drugs R D. 2017;17::97-101.

8. Kawai M, Yoshimura M, Harada M, et al. Determination of the B-type natriuretic peptide level as a criterion for abnormalities in Japanese individuals in routine clinical practice: the J-ABS Multi-Center Study (Japan Abnormal BNP Standard). Intern Med. 2013;52:171-7.

9. Shoji A, Yamanaka H, Kamatani N. A retrospective study of the relationship between serum urate level and recurrent attacks of gouty arthritis: evidence for reduction of recurrent gouty arthritis with antihyperuricemic therapy. Arthritis Rheum. 2004;51:321-5.

10. Imai E, Horio $\mathrm{M}$, Yamagata $\mathrm{K}$, et al. Slower decline of glomerular filtration rate in the Japanese general population: a longitudinal 10-year follow-up study. Hypertens Res. 2008;31:433 - 41.

11. Node K, Inoue T, Boyko V, et al. Long-term effects of peroxisome proliferator-activated receptor ligand bezafibrate on $\mathrm{N}$-terminal pro-B type natriuretic peptide in patients with advanced functional capacity impairment. Cardiovasc Diabetol. 2009;8:5.

12. Corretti MC, Anderson TJ, Benjamin EJ, et al. Guidelines for the ultrasound assessment of endothelial-dependent flow-mediated vasodilation of the brachial artery: a report of the International Brachial Artery Reactivity Task Force. J Am Coll Cardiol. 2002;39:257 - 65 .

13. Inoue $\mathrm{T}$, Matsuoka $\mathrm{H}$, Higashi $\mathrm{Y}$, et al. Flow-mediated vasodilation as a diagnostic modality for vascular failure. Hypertens Res. 2008;31:2105-13.

14. Matsubara J, Sugiyama S, Akiyama E, et al. Dipeptidyl peptidase-4 inhibitor, sitagliptin, improves endothelial dysfunction in association with its anti-inflammatory effects in patients with coronary artery disease and uncontrolled diabetes. Circulation. 2013;77:1337-44.

15. Kuki S, Matsuyama A, Masuno T, Hatashi Y. The clinical experience of topiroxostat in patients at high risk cardiovascular disease (in Japanese). Ther Res. 2016;37:49-57.

16. Givertz MM, Anstrom KJ, Redfield MM, et al. Effects of xanthine oxidase inhibition in hyperuricemic heart failure patients: the xanthine oxidase inhibition for hyperuricemic heart failure patients (EXACT-HF) study. Circulation. 2015;131:1741-4.

17. Kao MP, Ang DS, Gandy SJ, et al. Allopurinol benefits left ventricular mass and endothelial dysfunction in chronic kidney disease. J Am Soc Nephrol. 2011;22:1382-9.

18. Szwejkowski BR, Gandy SJ, Rekhraj S, et al. Allopurinol reduces left ventricular mass in patients with type 2 diabetes and left ventricular hypertrophy. J Am Coll Cardiol. 2013;62:2284-93.

19. Li X, Zhang J, Huang J, et al. A multicenter, randomized, doubleblind, parallel-group, placebo-controlled study of the effects of Qili Qiangxin capsules in patients with chronic heart failure. J Am Coll Cardiol. 2013;62:1065-72.

20. Sakai T, Tsutamoto T, Tsutsui T, Tanaka T, Ishikawa C, Horie M. Serum level of uric acid, partly secreted from the failing heart, is a prognostic marker in patients with congestive heart failure. Circ J. 2006;70:1006-11.

21. Krishnan E, Hariri A, dabbous O, Pandya BJ. Hyperuricemia and the echocardiographic measures of myocardial dysfunction. Congest Heart Fail. 2012;18:136 - 43.

22. Fujiyama Y, Yamauchi Y, Murase T, et al. Relationship between plasma xanthine oxidoreductase activity and left ventricular ejection fraction and hypertrophy among cardiac patients. PLos One. 2017;12:e0182699.

23. Erdogan D, Yayyar S, Uysal BA, et al. Effects of allopurinol on coronary microvascular and left ventricular function in 
patients with idiopathic dilated cardiomyopathy. Can J Cardiol. 2012;28:721-7.

24. Xiao J, Deng SB, She Q, et al. Allopurinol ameliorates cardiac function in non-hyperuricaemic patients with chronic heart failure. Eur Rev Med Pharmacol Sci. 2016;20:756-76.

25. Kamatani N, Fujimori S, Hada T, et al. An allopurinol-controlled, randomized, double-dummy, double-blind, parallel betweengroup, comparative study of febuxostat (TMX-67), a non-purineselective inhibitor of xanthine oxidase, in patients with hyperuricemia including those with gout in Japan: phase 3 clinical study. J Clin Rheumatol. 2011;17:S13-8.

26. Nomura J, Busso N, Ives A, et al. Xanthine oxidase inhibition by febuxostat attenuates experimental atherosclerosis in mice. Sci Rep. 2014;4:4554.

27. Oyama J, Tanaka A, Sato Y, et al. Rationale and design of a multicenter randomized study for evaluating vascular function under uric acid control using the xanthine oxidase inhibitor, febuxostat: the PRIZE study. Cardiovasc Diabetol. 2016;15:87.

28. Kawamorita Y, Shiraishi T, Tamura Y, et al. Renoprotective effect of topiroxostat via antioxidant activity in puromycin aminonucleoside nephrosis rats. Physiol Rep. 2017;5:e13358.

29. Yndestad A, Damås JK, Oie E, Ueland T, Gullestad L, Aukrust P. Systemic inflammation in heart failure-the whys and wherefores. Heart Fail Rev. 2006;11:83-92.
30. Ayoub KF, Pothineni NVK, Rutland J, Ding Z, Mehta JL. Immunity, inflammation, and oxidative stress in heart failure: emerging molecular targets. Cardiovasc Drugs Ther. 2017;31:593-608.

31. Shantsila E, Wrigley BJ, Blann AD, Gill PS, Lip GY. A contemporary view on endothelial function in heart failure. Eur J Heart Fail. 2012;14:873 - 81.

32. Schefold JC, Filippatos G, Hasenfuss G, Anker SD, von Haehling $S$. Heart failure and kidney dysfunction: epidemiology, mechanisms and management. Nat Rev Nephrol. 2016;12:610 - 23.

33. Masson S, Latini R, Anand IS, et al. Direct comparison of B-type natriuretic peptide (BNP) and amino-terminal proBNP in a large population of patients with chronic and symptomatic heart failure: the Valsartan Heart Failure (Val-HeFT) data. Clin Chem. 2006;52:1528-38.

34. Weber M, Hamm C. Role of B-type natriuretic peptide (BNP) and NT-proBNP in clinical routine. Heart. 2006;92:843-9.

35. Januzzi JL, Butler J, Jarolim P, et al. Effects of canagliflozin on cardiovascular biomarkers in older adults with type 2 diabetes. J Am Coll Cardiol. 2017;70:704 - 12.

\section{Affiliations}

\section{Masashi Sakuma ${ }^{1} \cdot$ Shigeru Toyoda $^{1} \cdot$ Takuo Arikawa $^{1} \cdot$ Yota Koyabu $^{1} \cdot$ Toru Kato $^{2} \cdot$ Taichi Adachi $^{3} \cdot$ Hideaki Suwa $^{3}$. Jun-ichi Narita ${ }^{4} \cdot$ Koetsu Anraku $^{5} \cdot$ Kimihiko Ishimura $^{5} \cdot$ Fumitake Yamauchi $^{6} \cdot$ Yasunori Sato $^{7} \cdot$ Teruo Inoue $^{1} \cdot$ For $^{3}$ Excited UA study Investigators}

\author{
Masashi Sakuma \\ masakuma@dokkyomed.ac.jp \\ Shigeru Toyoda \\ s-toyoda@dokkyomed.ac.jp \\ Takuo Arikawa \\ takuoari@dokkyomed.ac.jp \\ Yota Koyabu \\ left-pitch-robot@i.softbank.jp \\ Toru Kato \\ torumed3@gmail.com \\ Taichi Adachi \\ taiadachi@gmail.com \\ Hideaki Suwa \\ hideaki.suwa.wwl@gmail.com \\ Jun-ichi Narita \\ narita@cc9.ne.jp \\ Koetsu Anraku \\ kouetsu_a@ybb.ne.jp \\ Kimihiko Ishimura \\ ishimura@dokkyomed.ac.jp
}

Fumitake Yamauchi

yamauchi7758@yahoo.co.jp

Yasunori Sato

yasu@faculty.chiba-u.jp

1 Department of Cardiovascular Medicine, Dokkyo Medical University, 880 Kitakobayashi, Mibu, Tochigi 321-0293, Japan

2 Department of Clinical Research, National Hospital Organization Tochigi Medical Center, Utsunomiya, Japan

3 Department of Cardiology, National Hospital Organization Tochigi Medical Center, Utsunomiya, Japan

4 Department of Neurosurgery, Tochigi Medical Center Tochinoki, Tochigi, Japan

5 Department of Internal Medicine, Tochigi Medical Center Tochinoki, Tochigi, Japan

6 Department of Cardiology, Yuai Memorial Hospital, Koga, Japan

7 Department of Global Clinical Research, Graduate School of Medicine, Chiba University, Chiba, Japan 